\title{
Modified Method of Characteristics Variational Multiscale Finite Element Method for Time Dependent Navier-Stokes Problems*
}

\author{
Zhiyong $\mathrm{Si}^{a}$, Yunxia Wang ${ }^{b}$ and Xinlong Feng ${ }^{c}$ \\ ${ }^{a}$ School of Mathematics and Information Science, Henan Polytechnic \\ University \\ Jiaozuo 454003, P.R. China \\ ${ }^{b}$ School of Materials Science and Engineering, Henan Polytechnic University \\ Jiaozuo 454003, P.R. China \\ ${ }^{c}$ College of Mathematics and Systems Science, Xinjiang University \\ Urumqi 830046, P.R. China \\ E-mail(corresp.): sizhiyong@hpu.edu.cn \\ E-mail: wangyunxia@hpu.edu.cn \\ E-mail: fxlmath@gmail.com
}

Received March 6, 2015; revised August 26, 2015; published online September 15, 2015

\begin{abstract}
In this paper, a modified method of characteristics variational multiscale (MMOCVMS) finite element method is presented for the time dependent NavierStokes problems, which is leaded by combining the characteristics time discretization with the variational multiscale (VMS) finite element method in space. The theoretical analysis shows that this method has a good convergence property. In order to show the efficiency of the MMOCVMS finite element method, some numerical results of analytical solution problems are presented. First, we give some numerical results of lid-driven cavity flow with $R e=5000$ and 7500 as the time is sufficient long. From the numerical results, we can see that the steady state numerical solutions of the time-dependent Navier-Stokes equations are obtained. Then, we choose $R e=10000$, and we find that the steady state numerical solution is not stable from $t=200$ to 300. Moreover, we also investigate numerically the flow around a cylinder problems. The numerical results show that our method is highly efficient.
\end{abstract}

Keywords: modified method of characteristics, VMS finite element method, time dependent Navier-Stokes problems, characteristics-based method, numerical tests.

AMS Subject Classification: 76D05; 76M10; 65M60; 65M12.

* This work was supported in part by the NSF of China (Grant No. 11301156, 11401177 and 11271313). 


\section{Introduction}

In this paper, we consider the time-dependent Navier-Stokes problems

$$
\begin{cases}u_{t}-\nu \triangle u+(u \cdot \nabla) u+\nabla p=f, & x \in \Omega \times[0, T], \\ \nabla \cdot u=0, & x \in \Omega \times[0, T], \\ u(x, 0)=u_{0}(x), & x \in \Omega, \\ u(x, t)=0, & x \in \partial \Omega \times[0, T],\end{cases}
$$

where $\Omega$ is a bounded domain in $\mathbb{R}^{2}$ assumed to have a Lipschitz continuous boundary $\partial \Omega$. $u=\left(u_{1}(x, t), u_{2}(x, t)\right)^{T}$ represents the velocity vector, $p(x, t)$ represents the pressure, $f(x, t)$ is the body force, $\nu=1 / \operatorname{Re}$ the viscosity number, $R e$ is the Reynolds number.

Developing efficient finite element methods for the Stokes and Navier-Stokes equations is a key component in the incompressible flow simulation. There are some iterative methods for solving the stationary Navier-Stokes equations under some strong uniqueness conditions were presented by some authors [15, 18, 19]. The VMS method was first introduced by Hughes and his coworkers in $[22,23]$. The basic idea is splitting the solution into resolved and unresolved scale, representing the unresolved scales in terms of the resolved scales, and using this representation in the variational equation for the resolved scales. And there are many works devoted to this method, e.g. VMS method for the Navier-Stokes equations [26]; a two-level VMS method for convectiondominated convection-diffusion problems [29]; VMS methods for turbulent flow $[9,30,31]$; large-eddy simulation (LES) [24, 25,32]; subgrid-scale models for the incompressible flow $[22,44]$. In $[27,28]$, John et al. presented the error analysis of the two different kinds of VMS for the Navier-Stokes equations. The main difference is the definition of the large scales projection $\left(L^{2}\right.$-projection in [28] and elliptic projection in [27]). There is another class of VMS method which rely on a three-scale decomposition of the flow field into large, resolved small and unresolved scales [8].

In the characteristics method, which is a highly effective method for advection dominated problems, the hyperbolic part (the temporal and advection term) is treated by a characteristic tracking scheme. In 1982, Douglas and Russell [11] presented the modified method of characteristics finite element method (MMOCFEM) firstly. Russell [36] extended it to nonlinear coupled systems in two and three spatial dimensions. The second order in time method for linear convection diffusion problems had been given by Ewing and Russell [13]. A characteristics mixed finite element method for advection-dominated transport problems was presented by Arbogast [2] and for Navier-stokes equations by Buscagkia and Dari [7], respectively. In [34], a detail theory analysis for Navier-Stokes equations have been done by Pironneau, who obtained suboptimal convergence rates of the form $\mathcal{O}\left(h^{m}+\Delta t+h^{m+1} / \triangle t\right)$ and improved by Dawson et al [10]. In [4], Boukir et al. presented a second-order time scheme based on the characteristics method and spatial discretization of finite element type for the incompressible Navier-Stokes equations. An optimal error estimate for the Lagrange-Galerkin mixed finite element approximation of the Navier-Stokes equations was given by Süli in [43]. In $[38,39]$, one order and 
second order MMOC mixed defect-correction finite element methods for timedependent Navier-Stokes problems were given. In [41], second order in time MMOC variational multiscale finite element method for the time-dependent Navier-Stokes equation was shown. In [40], we presented modified characteristics gauge-Uzawa finite element method for the conduction-convection equation. In [42], the modified characteristics finite element method for the NavierStokes/Darcy problem was shown.

In this paper, we present a modified method of characteristics VMS finite element method based on the $L^{2}$ projection for the time dependent NavierStokes equations. In our method, the hyperbolic part (the temporal and advection term) is treated by a characteristic tracking scheme. The VMS based on projection finite element method is used for spatial discretization. The error analysis shows that this method has a good convergence property. In order to show the efficiency of the MMOCVMS finite element method, we firstly present some numerical results of analytical solution problems. The numerical results show that the convergence rates are $\mathcal{O}\left(h^{3}\right)$ in the $L^{2}$-norm for $u, \mathcal{O}\left(h^{2}\right)$ in the semi $H^{1}$-norm for $u$ and $\mathcal{O}\left(h^{2}\right)$ in the $L^{2}$-norm for $p$ by using the Taylor-Hood element, which agrees very well with our theoretical results. Then, some numerical results of the lid-driven cavity flow with $R e=5000$ and 7500 are given. We present the numerical results as the time are sufficient long enough, then the solution of the Navier-Stokes should approximate the solution of the steady state cases. From the numerical results, we can see that a steady state numerical solutions of the time-dependent Navier-Stokes equations are obtained. And the numerical solutions are in good agreement with that of the steady NavierStokes equations shown by Ghia et al. [14] and Erturk et al. [12]. Finally, we present some numerical results for $R e=10000$. It shows that the solution is mainly periodic with small variations in the amplitude of the time evolution at the monitoring points as the time is sufficient long. And the phase portraits of the monitoring points show that the variations in amplitude yield a solution which is quasi-periodic. Furthermore, we present numerical simulations for the two-dimensional fluid flow around a cylinder. It is observed from these numerical results that the scheme can result in good accuracy, which shows that this method is highly efficient.

The rest of this paper is organized as follows. In Section 2, the functional settings of the Navier-Stokes equations are presented. In Section 3 the MMOCVMS finite element methods is proposed. The following Section 4 we derive optimal error estimate for the new algorithm. Numerical experiments will be carried out in Section 5 and some concluding remarks will be given in the final section.

\section{Functional settings of the Navier-Stokes equations}

In this section, we aim to describe some notations and results which will be frequently used in this paper. Firstly we introduce the standard Sobolev spaces

$$
H^{n}(0, T ; \Phi)=\left\{v \in \Phi ; \sum_{0 \leq i \leq n} \int_{0}^{T}\left(\left\|\frac{\partial^{i} v}{\partial t^{i}}\right\|_{\Phi}\right)^{2} d t<+\infty\right\},
$$


equipped with the norm

$$
\|v\|_{H^{n}(\Phi)}=\left[\sum_{0 \leq i \leq n} \int_{0}^{T}\left(\left\|\frac{\partial^{i} v}{\partial t^{i}}\right\|_{\Phi}\right)^{2} d t\right]^{\frac{1}{2}} .
$$

Here $\Phi$ denotes a Hilbert space with the norm $\|\cdot\|_{\Phi}$. Especially, when $n=0$ we note

$$
\|v\|_{L^{2}(\Phi)}=\left(\int_{0}^{T}\|v\|_{\Phi}^{2} d t\right)^{\frac{1}{2}}
$$

And, we define

$$
L^{\infty}(0, T ; \Phi)=\left\{v \in \Phi ; \text { ess } \sup _{0 \leq t \leq T}\|v\|_{\Phi}<+\infty\right\}
$$

with the norm

$$
\|v\|_{L^{\infty}(\Phi)}=\operatorname{ess} \sup _{0 \leq t \leq T}\|v\|_{\Phi}
$$

For the mathematical setting of the Navier-Stokes problems (1.1), we introduce the following Hilbert spaces

$$
\begin{aligned}
X & :=H_{0}^{1}(\Omega)^{2}, \quad M:=L_{0}^{2}(\Omega)=\left\{\varphi \in L^{2}(\Omega) ; \int_{\Omega} \varphi d x=0\right\}, \\
V & :=\{v \in X ;(\nabla \cdot v, q)=0 \forall q \in M\} .
\end{aligned}
$$

The following assumptions and results are recalled (see [18]).

$\left(\mathbf{A}_{\mathbf{1}}\right)$ There exists a positive constant $C_{0}$ which only depends on $\Omega$, such that

$$
\|u\|_{0} \leq C_{0}\|\nabla u\|_{0} \forall u \in X
$$

$\left(\mathbf{A}_{2}\right)$ Assume that $\Omega$ is smooth, hence the unique solution $(v, q) \in(X, M)$ of the steady Stokes problem

$$
-\triangle v+\nabla q=g, \quad \nabla \cdot v=0, \text { in } \Omega,\left.\quad v\right|_{\partial \Omega}=0
$$

for any prescribed $g \in L^{2}(\Omega)^{2}$ exits and satisfies

$$
\|v\|_{2}+\|q\|_{1} \leq c\|g\|_{0}
$$

where $c>0$ is a generic constant depending on $\Omega$, which may stand for different values at its different occurrences. $\|\cdot\|_{2}$ means the $H^{2}$-norm, $\|\cdot\|_{1}$ means the $H^{1}-$ norm, and $\|\cdot\|_{0}$ means the $L^{2}-$ norm, respectively.

For simplicity, we assume that the time-dependent Navier-Stokes system (1.1) has a unique solution $(u, p)$ satisfying

$$
\begin{aligned}
\left\|u_{0}\right\|_{H^{r+1}} & +\|u\|_{L^{\infty}\left(I ; H^{r+1}\right)}+\left\|u_{t}\right\|_{L^{2}\left(I ; H^{r+1}\right)}+\left\|u_{t t}\right\|_{L^{2}\left(I ; L^{2}\right)} \\
& +\|p\|_{L^{\infty}\left(I ; H^{r}\right)}+\left\|p_{t}\right\|_{L^{2}\left(I ; H^{r}\right)} \leq C
\end{aligned}
$$

with $I=(0, T)$ and $r \geq 2$. 


\section{MMOCVMS finite element methods}

\subsection{MMOC-mixed finite element methods for the time-dependent Navier-Stokes equations}

For each positive integer $N$, let $\left\{\mathcal{J}_{n}: 1 \leq n \leq N\right\}$ be a partition of $[0, T]$ into subintervals $\mathcal{J}_{n}=\left(t_{n-1}, t_{n}\right]$, with $t_{n}=n \Delta t, \triangle t=T / N$. Set $u^{n}=u\left(\cdot, t_{n}\right)$ be the $u(x, t)$ at $t=t_{n}$. The characteristic trace-back along the field $u^{n-1}$ of a point $x \in \Omega$ at time $t_{n}$ to $t_{n-1}$ is approximately $\bar{x}\left(x, t_{n-1}\right)=x-\Delta t u^{n-1}$.

Consequently, the hyperbolic part in the first equation of (1.1) at time $t_{n}$ is approximated by

$$
u_{t}+u^{n-1} \cdot \nabla u^{n} \approx \frac{u^{n}-\bar{u}^{n-1}}{\triangle t},
$$

where

$$
\bar{w}= \begin{cases}w(\bar{x}), & \bar{x}=x-\triangle t u^{n-1} \in \Omega \\ 0, & \text { otherwise }\end{cases}
$$

for any function $w$.

Let $\Im_{h}$ be a quasi-uniform partition of $\bar{\Omega}$ into non-overlapping triangles, indexed by a parameter $h=\max _{K \in \Im_{h}}\left\{h_{K} ; h_{K}=\operatorname{diam}(K)\right\}$. We introduce the finite element subspace $X_{h} \subset X, M_{h} \subset M$ as follows

$$
\begin{aligned}
& X_{h}=\left\{v_{h} \in X \cap C^{0}(\bar{\Omega})^{2} ;\left.v_{h}\right|_{K} \in P_{\ell}(K)^{2} \forall K \in \Im_{h}\right\}, \\
& M_{h}=\left\{q_{h} \in M \cap C^{0}(\bar{\Omega}) ;\left.q_{h}\right|_{K} \in P_{k}(K) \forall K \in \Im_{h}\right\},
\end{aligned}
$$

where $P_{\ell}(K)$ is the space of polynomials of degree $\ell$ on $K . \ell \geq 1, k \geq 1$ are two integers. Assume that $\left(X_{h}, M_{h}\right)$ satisfies the discrete LBB condition

$$
\sup _{v_{h} \in X_{h}} \frac{d\left(\varphi_{h}, v_{h}\right)}{\left\|\nabla v_{h}\right\|_{0}} \geq \beta\left\|\varphi_{h}\right\|_{0} \forall \varphi_{h} \in M_{h},
$$

where $d(\varphi, v)=(\varphi, \nabla \cdot v), \beta>0$ is a constant independent of $h$. In this paper, $V_{h}$ denotes the kernel of the discrete divergence operator

$$
V_{h}=\left\{v_{h} \in X_{h} ;\left(q_{h}, \nabla \cdot v_{h}\right)=0 \forall q_{h} \in M_{h}\right\}
$$

With the previous notations, we present the MMOC finite element method for the Navier-Stokes (1.1): find $\left(u^{n}, p^{n}\right):\left\{t_{1}, \ldots, t_{N}\right\} \rightarrow V_{h} \times M_{h}$ such that

$$
\begin{array}{r}
\left(\frac{u_{h}^{n}-\check{u}_{h}^{n-1}}{\Delta t}, v_{h}\right)+\nu a\left(u_{h}^{n}, v_{h}\right)-d\left(p_{h}^{n}, v_{h}\right)+ \\
\quad d\left(\varphi_{h}, u_{h}^{n}\right)=\left(f^{n}, v_{h}\right) \\
\forall v_{h} \in X_{h}, \varphi_{h} \in M_{h},
\end{array}
$$

where $a(u, v)=(\nabla u, \nabla v), f^{n}=f\left(t_{n}\right)$,

$$
\check{u}_{h}^{n-1}= \begin{cases}u_{h}^{n-1}(\check{x}), & \check{x}=x-u_{h}^{n-1} \triangle t \in \Omega, \\ 0, & \text { otherwise. }\end{cases}
$$




\subsection{The VMS finite element method and MMOCVMS finite ele- ment method for the time-dependent Navier-Stokes equations}

For the VMS finite element method, a large scale space $L_{H} \subset L=\{\mathbb{L} \in$ $\left.\left(L^{2}(\Omega)\right)^{d \times d}, \mathbb{L}=\mathbb{L}^{T}\right\}$ and a so-called turbulent viscosity $\nu_{T} \geq 0$ are introduced. The semi-discrete problems reads as follows (please see [31]): Find $u_{h}:[0, T] \rightarrow$ $v_{h}, p_{h}:[0, T] \rightarrow M_{h}$ and $\mathbb{G}_{H}:[0, T] \rightarrow L_{H}$ satisfying

$$
\begin{gathered}
\left(u_{h t}, v_{h}\right)+\left(\nu+\nu_{T}\right) a\left(u_{h}, v_{h}\right)-d\left(p_{h}, v_{h}\right)+d\left(\varphi_{h}, u_{h}\right)+b\left(u_{h}, u_{h}, v_{h}\right) \\
-\left(\nu_{T} \mathbb{G}_{H}, \nabla v_{h}\right)=\left(f, v_{h}\right) \quad \forall v_{h} \in X_{h}, \varphi_{h} \in M_{h}, \\
\left(\mathbb{G}_{H}-\nabla u_{h}, \mathbb{L}_{h}\right)=0 \quad \forall \mathbb{L}_{H} \in L_{H},
\end{gathered}
$$

where $u_{h}(x, 0)=u_{h}^{0} \in V_{h}$ is a discretely divergence free approximation of $u_{0}$,

$$
b(u, v, w)=\frac{1}{2}\left[\int_{\Omega} \sum_{i, k=1}^{2} u_{i} \frac{\partial v_{k}}{\partial x_{i}} w_{k}-\sum_{i, k=1}^{2} u_{i} \frac{\partial w_{k}}{\partial x_{i}} v_{k} d x\right] \forall u, v, w \in X_{h} .
$$

Let $P_{L_{H}}: L \rightarrow L_{H}$ for all $v \in V$, with

$$
\left(P_{L_{H}} \nabla v-\nabla v, \mathbb{L}_{H}\right)=0 \forall \mathbb{L}_{H} \in L_{H}
$$

denote the $L^{2}$-projection from $L$ onto $L_{H}$. Then, $\mathbb{G}_{H}=P_{L_{H}} \nabla u_{h}$ in (3.2). Since $P_{L_{H}}$ is an $L^{2}$-projection, it follows for $v \in V$ and $\|\nabla v\|_{0}>0$,

$$
\begin{aligned}
\nu_{T}\left\|\left(I-P_{L_{H}}\right) \nabla v\right\|_{0}^{2} & =\nu_{T}\left(\|\nabla v\|_{0}^{2}-\left\|P_{L_{H}} \nabla v\right\|_{0}^{2}\right) \\
& =\nu_{T}\left(1-\frac{\left\|P_{L_{H}} \nabla v\right\|_{0}^{2}}{\|\nabla v\|_{0}^{2}}\right)\|\nabla v\|_{0}^{2} \equiv \nu_{a d d}\|\nabla v\|_{0}^{2}
\end{aligned}
$$

By $0 \leq\left\|P_{L_{H}} \nabla v\right\|_{0} \leq\|\nabla v\|_{0}$, we get $0 \leq \nu_{a d d} \leq \nu_{T}$. By straightforward calculation, we deduce

$$
\left(\nu_{T} \nabla u_{h}, \nabla v_{h}\right)-\left(\nu_{T} P_{L_{H}} \nabla u_{h}, \nabla v_{h}\right)=\left(\nu_{T}\left(I-P_{L_{H}}\right) \nabla u_{h},\left(I-P_{L_{H}}\right) \nabla v_{h}\right) .
$$

Hence, system (3.2) can be reformulated as: Find $u_{h}:[0, T] \rightarrow X_{h}, p_{h}$ : $[0, T] \rightarrow M_{h}$ satisfying

$$
\begin{aligned}
\left(u_{h t}, v_{h}\right)+\nu a\left(u_{h}, v_{h}\right) \quad & -d\left(p_{h}, v_{h}\right)+d\left(\varphi_{h}, u_{h}\right)+b\left(u_{h}, u_{h}, v_{h}\right) \\
& +\left(\nu_{T}\left(I-P_{L_{H}}\right) \nabla u_{h},\left(I-P_{L_{H}}\right) \nabla v_{h}\right) \\
& =\left(f, v_{h}\right) \quad \forall v_{h} \in X_{h}, \varphi_{h} \in M_{h} .
\end{aligned}
$$

The MMOC time discretization, combined with the VMS finite element method in space, leads to the following MMOCVMS finite element method.

Find $\left(u_{h}^{n}, p_{h}^{n}\right) \in\left(X_{h} \cap V_{h}\right) \times M_{h}$, such that

$$
\begin{gathered}
\left(\frac{u_{h}^{n}-\hat{u}_{h}^{n-1}}{\Delta t}, v_{h}\right)+\left(\nu+\nu_{T}\right) a\left(u_{h}^{n}, v_{h}\right)-d\left(p_{h}^{n}, v_{h}\right)+d\left(\varphi_{h}, u_{h}^{n}\right) \\
-\left(\nu_{T} \mathbb{G}_{H}^{n}, \nabla v_{h}\right)=\left(f^{n}, v_{h}\right) \quad \forall v_{h} \in X_{h}, \varphi_{h} \in M_{h}, \\
\left(\mathbb{G}_{H}^{n}-\nabla u_{h}^{n}, \mathbb{L}_{H}\right)=0 \quad \forall \mathbb{L}_{H} \in L_{H},
\end{gathered}
$$


where

$$
\hat{u}_{h}^{n-1}= \begin{cases}u_{h}^{n-1}(\hat{x}), & \hat{x}=x-u_{h}^{n-1} \tau \in \Omega, \\ 0, & \text { otherwise. }\end{cases}
$$

Using (3.4), (3.5) can be reformulated as

$$
\begin{aligned}
& \left(\frac{u_{h}^{n}-\hat{u}_{h}^{n-1}}{\triangle t}, v_{h}\right)+\nu a\left(u_{h}^{n}, v_{h}\right)-d\left(p_{h}^{n}, v_{h}\right)+d\left(\varphi_{h}, u_{h}^{n}\right) \\
& \quad+\left(\nu_{T}\left(I-P_{L_{H}}\right) \nabla u_{h},\left(I-P_{L_{H}}\right) \nabla v_{h}\right)=\left(f^{n}, v_{h}\right) \quad \forall v_{h} \in X_{h}, \varphi_{h} \in M_{h} .
\end{aligned}
$$

Remark 1. Since $L_{H}$ has been distinguish between resolved small scales and large scales, with $L_{H}$ representing the large scales, it must be in some sense a coarse finite element space. One way of achieving this is choosing it to be a lower order finite element space than $V_{h}$ on the same grid (e.g. $L_{H}=$ $\mathbb{D}\left(V_{h}\right), \mathbb{D}(v)=\left(\nabla v+\nabla v^{T}\right) / 2$, see [27] for details). The other way is defining $L_{H}$ on a coarser grid. This two methods are called one-level and two-level projection-based FEVMS methods respectively (see $[29,31]$ ). The so-called turbulent viscosity $\nu_{T}$ can be chosen in many ways. In this paper we choose as follows (Smagorinsky-type [26]) $\nu_{T}=\delta h^{2}\left\|\nabla u_{h}^{n}\right\|_{0}$, where $\delta$ is a suitable constant.

Remark 2. [35, 44] If $L_{H}=\left\{v_{h} \in\left(C^{0}(\Omega)\right)^{2} \cap X:\left.v_{h}\right|_{K} \in\left(P_{k}(K)\right)^{2} \forall K \in \Im_{h}\right\}$, then we have the following properties

$$
\begin{aligned}
\left\|P_{L_{H}} v\right\|_{1} & \leq C\|v\|_{1} \forall v \in X,\left\|\nabla\left(I-P_{L_{H}}\right) v\right\|_{0} \leq C\|\nabla v\|_{0} \forall v \in X, \\
\left\|v-P_{L_{H}} v\right\|_{1} & \leq C h^{l}\|v\|_{l+1}, 1 \leq l \leq k \forall v \in H^{l+1}(\Omega)^{2} \cap X .
\end{aligned}
$$

\section{Error estimate}

In order to obtain the error analysis, we give some lemmas firstly.

Lemma 1. [39] Let

$$
e(x, n)=\frac{u^{n}(x)-\bar{u}^{n-1}(x)}{\triangle t}-\left(\frac{\partial u}{\partial t}\left(x, t_{n}\right)+u^{n}(x) \nabla u^{n}(x)\right)
$$

and $\tau>0$ such that $u \in \mathcal{C}^{4}\left([\tau, T] ; H^{3}(\Omega)^{2}\right)$. For $t_{n}>\tau$, we have

$$
e(x, n)=-\triangle t\left(\frac{1}{2} \frac{d^{2} g_{x}^{n}}{d t^{2}}+\frac{\partial u}{\partial t} \cdot \nabla u\left(x, t_{n}\right)\right)+O\left(\triangle t^{2}\right),
$$

where $g_{x}^{n}(t)=u\left(x-\left(t_{n}-t\right) u^{n-1}, t\right), u^{n}(x)=u\left(x, t_{n}\right)$.

Then, we define the following Galerkin projection

$$
\left(R_{h}, Q_{h}\right)=\left(R_{h}(u, p), Q_{h}(u, p)\right):(X, M) \rightarrow\left(X_{h}, M_{h}\right),
$$

such that

$$
\begin{aligned}
\nu a\left(R_{h}-u, v_{h}\right)- & d\left(Q_{h}-p, v_{h}\right)+d\left(\varphi_{h}, R_{h}-u\right)=0 \\
& \forall(u, p) \in(X, M),\left(v_{h}, \varphi_{h}\right) \in\left(X_{h}, M_{h}\right) .
\end{aligned}
$$


Lemma 2. [20,33] The Galerkin projection $\left(R_{h}, Q_{h}\right)$ satisfies

$$
\begin{aligned}
\left\|R_{h}-u\right\|_{0} & +h\left(\left\|\nabla\left(R_{h}-u\right)\right\|_{0}+\left\|Q_{h}-p\right\|_{0}\right) \\
& \leq C h^{k+1}\left(\nu\|u\|_{k+1}+\|p\|_{k}\right), \quad k=1,2 .
\end{aligned}
$$

Lemma 3. [1,3] The Galerkin projection $\left(R_{h}, Q_{h}\right)$ satisfies

$$
\left\|R_{h}\right\|_{\infty} \leq C\left(\|u\|_{\infty}+h^{k}\|u\|_{H^{k+1}(\Omega)}\right) .
$$

\subsection{Error estimate for the velocity}

Lemma 4. Let $u_{h}^{n}$ be defined by (3.6). If $\triangle t \leq \min \left\{1 /\left(2 L_{n}\right), h\right\}, L_{n}=$ $\max _{1 \leq l \leq n-1}\left\|\nabla u_{h}^{l}\right\|_{\infty}$, for all $2 \leq n \leq N$ we have

$$
\begin{aligned}
& \left\|\xi_{h}^{n}\right\|_{0}^{2}+\nu \triangle t \sum_{i=1}^{n}\left\|\nabla \xi_{h}^{i}\right\|_{0}^{2} \\
& \quad \leq C \exp (C T)\left(\Delta t^{2}+h^{2 k+2}+\nu_{a d d} h^{2 k}+\nu_{T}\left\|\left(I-P_{L_{H}}\right) \nabla u^{n}\right\|_{0}^{2}\right), \\
& \left\|u_{h}^{n}\right\|_{\infty}<+\infty, \quad \triangle t\left\|u_{h}^{n}\right\|_{W^{1, \infty}} \leq 1 / 4,
\end{aligned}
$$

where $\xi_{h}^{n}=u_{h}^{n}-R_{h}^{n}, C$ is a positive constant independent of $\triangle t$ and $h$.

Remark 3. Define $\hat{\mathcal{X}}_{x}^{n}(t)=x-\left(t_{n}-t\right) u_{h}^{n-1} \forall t \in\left[t^{n-1}, t^{n}\right], 2 \leq n \leq N$. Since $X_{h}$ is a subset of $W^{1, \infty}(\Omega)$, under the condition $\triangle t \leq \frac{1}{2 L_{n}}$ on the time step it is an easy matter to verify that this mapping has a positive Jacobian, since $u_{h}^{l}$ vanishes on $\partial \Omega$; this mapping is one-to-one and this it is a change of variables from $\Omega$ onto $\Omega$. This yields for any positive function $\phi$ on $\Omega$ the estimate (please see [4] for details)

$$
\int_{\Omega} \phi\left(\hat{\mathcal{X}}_{h}^{n}(t)\right) d x \leq C \int_{\Omega} \phi(x) d x .
$$

Remark 4. Here, we assume that the initial condition $u_{0}$ has a strong regularity. Or there are some restriction on the time step $\triangle t[17]$.

Proof. We prove this lemma by induction. By the definition of $\hat{x}$, we can see that (4.5) and (4.4) hold for $n=0$. We assume that (4.5) and (4.4) hold for $1 \leq n \leq l-1$, then $L_{n}<+\infty$. Now we will prove them hold for $n=l$.

Letting $\varphi_{h}=0$ in (3.6) and using the definition of $V_{h}$, we have

$$
\begin{aligned}
& \left(\frac{u_{h}^{l}-\hat{u}_{h}^{l-1}}{\Delta t}, v_{h}\right)+\nu a\left(u_{h}^{l}, v_{h}\right) \\
& +\nu_{T}\left(\left(I-P_{L_{H}}\right) \nabla u_{h}^{l},\left(I-P_{L_{H}}\right) \nabla v_{h}\right)=\left(f^{l}, v_{h}\right) \forall v_{h} \in V_{h} .
\end{aligned}
$$

Subtracting $\left(\left(R_{h}^{l}-\hat{R}_{h}^{l-1}\right) / \triangle t, v_{h}\right)+\nu a\left(R_{h}^{l}, v_{h}\right)$ from both sides of (4.6), we get

$$
\begin{gathered}
\left(\frac{\left(u_{h}^{l}-R_{h}^{l}\right)-\left(\hat{u}_{h}^{l-1}-\hat{R}_{h}^{l-1}\right)}{\triangle t}, v_{h}\right)+\nu a\left(u_{h}^{l}-R_{h}^{l}, v_{h}\right) \\
+\nu_{T}\left(\left(I-P_{L_{H}}\right) \nabla u_{h}^{l},\left(I-P_{L_{H}}\right) \nabla v_{h}\right) \\
=\left(f^{l}, v_{h}\right)-\left(\frac{R_{h}^{l}-\hat{R}_{h}^{l-1}}{\triangle t}, v_{h}\right)-\nu a\left(R_{h}^{l}, v_{h}\right) .
\end{gathered}
$$


Define $\eta^{l}=u^{l}-R_{h}^{l}$, we arrive at

$$
\begin{aligned}
& \left(\frac{\xi_{h}^{l}-\xi_{h}^{l-1}}{\triangle t}, v_{h}\right)+\nu a\left(\xi_{h}^{l}, v_{h}\right)+\nu_{T}\left(\left(I-P_{L_{H}}\right) \nabla \xi_{h}^{l},\left(I-P_{L_{H}}\right) \nabla v_{h}\right) \\
& =-\left(\frac{u^{l}-\bar{u}^{l-1}}{\triangle t}-\nu \Delta u^{l}+\nabla p^{l}-f^{l}, v_{h}\right)+\left(\frac{\hat{u}^{l-1}-\bar{u}^{l-1}}{\triangle t}, v_{h}\right) \\
& \quad+\left(\frac{\eta^{l}-\hat{\eta}^{l-1}}{\triangle t}, v_{h}\right)+\left(\frac{\hat{\xi}_{h}^{l-1}-\xi_{h}^{l-1}}{\triangle t}, v_{h}\right) \\
& \quad+\nu_{T}\left(\left(I-P_{L_{H}}\right) \nabla \eta_{h}^{l},\left(I-P_{L_{H}}\right) \nabla v_{h}\right)+\nu_{T}\left(\left(I-P_{L_{H}}\right) \nabla u^{l},\left(I-P_{L_{H}}\right) \nabla v_{h}\right) \\
& \quad+\left(\nabla p^{l}, v_{h}\right)+\nu a\left(u^{l}-R_{h}^{l}, v_{h}\right) \\
& =\sum_{i=1}^{6} \Pi_{i}+d\left(Q_{h}^{l}-p^{l}, v_{h}\right)+\nu a\left(u^{l}-R_{h}^{l}, v_{h}\right)=\sum_{i=1}^{6} \Pi_{i},
\end{aligned}
$$

where $\Pi_{i}$ denotes the $i$-th term of the right-hand side of (4.8).

Next we give the estimate for each term. By the definition of $\hat{x}$ and $\bar{x}$, we arrive at

$$
\hat{x}\left(x, t_{l-1}\right)-\bar{x}\left(x, t_{l-1}\right)=\left(u_{h}^{l-1}-u^{l-1}\right) \triangle t .
$$

Using Taylor formula, we obtain

$$
\begin{aligned}
\left\|\hat{u}^{l-1}-\bar{u}^{l-1}\right\|_{0} & =\left\|u^{l-1}(\hat{x})-u^{l-1}(\bar{x})\right\|_{0} \\
& \leq \triangle t\left\|\nabla u^{l-1}\right\|_{\infty}\left\|u_{h}^{l-1}-u^{l-1}\right\|_{0} \\
& \leq \triangle t\left\|\nabla u^{l-1}\right\|_{\infty}\left(\left\|u_{h}^{l-1}-R_{h}^{l-1}\right\|_{0}+\left\|R_{h}^{l-1}-u^{l-1}\right\|_{0}\right) .
\end{aligned}
$$

Therefore, we get

$$
\begin{aligned}
\frac{1}{\triangle t}\left\|\hat{u}^{l-1}-\bar{u}^{l-1}\right\|_{0} & \leq\left\|\nabla u^{l-1}\right\|_{\infty}\left(\left\|u^{l-1}-R_{h}^{l-1}\right\|_{0}+\left\|R_{h}^{l-1}-u_{h}^{l-1}\right\|_{0}\right) \\
& \leq C\left(h^{k+1}+\left\|\xi_{h}^{l-1}\right\|_{0}\right) .
\end{aligned}
$$

Now, we estimate the bound of $\left\|\frac{\eta^{l}-\hat{\eta}^{l-1}}{\triangle t}\right\|_{0}$. By Cauchy-Schwarz inequality, we get

$$
\begin{aligned}
& \left\|\eta^{l}-\eta^{l-1}\right\|_{0}=\left(\int_{\Omega}\left(\eta^{l}-\eta^{l-1}\right)^{2} d x\right)^{\frac{1}{2}}=\left(\int_{\Omega}\left|\int_{t_{l-1}}^{t_{l}} \frac{\partial \eta}{\partial t}(x, \theta) d \theta\right|^{2} d x\right)^{\frac{1}{2}} \\
& \leq \sqrt{\triangle t}\left(\int_{\Omega} \int_{t_{l-1}}^{t_{l}}\left|\frac{\partial \eta}{\partial t}(x, \theta)\right|^{2} d \theta d x\right)^{\frac{1}{2}} \leq \sqrt{\triangle t}\left\|\frac{\partial \eta}{\partial t}\right\|_{L^{2}\left(\left[t_{l-1}, t_{l}\right]: L^{2}(\Omega)\right)}
\end{aligned}
$$

By the definition of $\hat{\mathcal{X}}_{x}^{l}\left(t_{l-1}\right)$, we have

$$
J\left(\hat{\mathcal{X}}_{x}^{l}\left(t_{l-1}\right)\right)=\left(\begin{array}{cc}
1-\partial_{x} u_{h 1}^{l-1} \triangle t & -\partial_{y} u_{h 1}^{l-1} \triangle t \\
-\partial_{x} u_{h 2}^{l-1} \triangle t & 1-\partial_{y} u_{h 2}^{l-1} \triangle t
\end{array}\right) .
$$


Hence, $\operatorname{det} J\left(\hat{\mathcal{X}}_{x}^{l}\left(t_{l-1}\right)\right)=1+\mathcal{O}(\triangle t)$. Then, we deduce

$$
\begin{aligned}
& \left\|\eta^{l-1}-\hat{\eta}^{l-1}\right\|_{-1}=\sup _{v \in V}\left(\|\nabla v\|_{0}^{-1}\left(\eta^{l-1}-\hat{\eta}^{l-1}, v\right)\right)=\sup _{v \in V}\left[\|\nabla v\|_{0}^{-1}\right. \\
& \left.\times\left(\int_{\Omega} \eta^{l-1}(x) v(x) d x-\int_{\Omega} \eta^{l-1}(z) v\left(\hat{\mathcal{X}}_{x}^{n}\left(t_{l-1}\right)^{-1}\right)\left(1+\mathcal{O}\left(\triangle t^{2}\right)\right) d z\right)\right] \\
& \leq \sup _{v \in V}\left(\|\nabla v\|_{0}^{-1} \int_{\Omega} \eta^{l-1}(x)\left(v(x)-v\left(\hat{\mathcal{X}}_{x}^{l}\left(t_{l-1}\right)^{-1}\right)\right) d x\right) \\
& \left.+\sup _{v \in V}\left(C \triangle t^{2}\|\nabla v\|_{0}^{-1} \int_{\Omega} \eta^{l-1}(z) v\left(\hat{\mathcal{X}}_{x}^{l}\left(t_{l-1}\right)^{-1}\right)\right) d z\right) .
\end{aligned}
$$

Let $G(x)=x-\hat{\mathcal{X}}_{x}^{l}\left(t_{l-1}\right)^{-1}$, then $|G(x)| \leq C \triangle t$, and

$$
\left\|v(x)-v\left(\hat{\mathcal{X}}_{x}^{l}\left(t_{l-1}\right)^{-1}\right)\right\|_{0}^{2} \leq \int_{\Omega}\left(\int_{t_{l-1}}^{t_{l}} \frac{d}{d t} v\left(\hat{\mathcal{X}}_{x}^{l}(t)^{-1}\right) d t\right)^{2} d x \leq C \Delta t^{2}\|\nabla v\|_{0}^{2}
$$

Similarly, we have

$$
\left\|v\left(\hat{\mathcal{X}}_{x}^{l}\left(t_{l-1}\right)^{-1}\right)\right\| \leq(1+C \triangle t)\|v\|_{0}^{2} .
$$

Then, we deduce

$$
\left\|\eta^{l-1}-\hat{\eta}^{l-1}\right\|_{-1} \leq C \triangle t\left\|\eta^{l-1}\right\|_{0}
$$

By (4.11) and (4.13), we get

$$
\left\|\frac{\eta^{l}-\hat{\eta}^{l-1}}{\triangle t}\right\|_{-1} \leq C h^{k+1}\left\|u_{h}^{l-1}\right\|_{\infty}+C \frac{h^{k+1}}{\sqrt{\triangle t}}\left\|\frac{\partial u}{\partial t}\right\|_{L^{2}\left(t_{l-1}, t_{l} ; H^{k}(\Omega)\right)} .
$$

Similarly, we obtain

$$
\left\|\frac{\xi_{h}^{l-1}-\hat{\xi}_{h}^{l-1}}{\triangle t}\right\|_{-1} \leq C\left\|\xi_{h}^{l-1}\right\|_{0} .
$$

Setting $v_{h}=\xi_{h}^{l}$ in (4.9), we obtain

$$
\begin{aligned}
& \frac{\left\|\xi_{h}^{l}\right\|_{0}^{2}-\left\|\xi_{h}^{l-1}\right\|_{0}^{2}}{2 \triangle t}+\left(\nu+\nu_{a d d}\right) a\left(\xi_{h}^{l}, \xi_{h}^{l}\right) \\
& \leq\left|\left(\frac{u^{l}-\bar{u}_{h}^{l-1}}{\triangle t}-\nu \triangle u^{l}+\nabla p^{l}-f^{l}, \xi_{h}^{l}\right)\right|+\left|\left(\frac{\eta^{l}-\hat{\eta}_{h}^{l-1}}{\triangle t}, \xi_{h}^{l}\right)\right| \\
& +\left|\left(\frac{\hat{u}^{l-1}-\bar{u}^{l-1}}{\triangle t}, \xi_{h}^{l}\right)\right|+\left|\left(\frac{\hat{\xi}_{h}^{l-1}-\xi_{h}^{l-1}}{\triangle t}, \xi_{h}^{l}\right)\right| \\
& +\left|\nu_{T}\left(\left(I-P_{L_{H}}\right) \nabla \eta_{h}^{n},\left(I-P_{L_{H}}\right) \nabla \xi_{h}^{l}\right)\right|+\left|\nu_{T}\left(\left(I-P_{L_{H}}\right) \nabla u^{l},\left(I-P_{L_{H}}\right) \nabla \xi_{h}^{l}\right)\right| .
\end{aligned}
$$


Using Cauchy-Schwarz and Young's inequalities, we deduce

$$
\begin{aligned}
& \frac{\left\|\xi_{h}^{l}\right\|_{0}^{2}-\left\|\xi_{h}^{l-1}\right\|_{0}^{2}}{2 \Delta t}+\left(\nu+\nu_{a d d}\right)\left\|\nabla \xi_{h}^{l}\right\|_{0}^{2} \\
& \leq C\left\|\frac{u^{l}-\bar{u}_{h}^{l-1}}{\Delta t}-\nu \Delta u^{l}+\nabla p^{l}-f^{l}\right\|_{0}\left\|\nabla \xi_{h}^{l}\right\|_{0}+C\left\|\frac{\eta^{l}-\hat{\eta}_{h}^{l-1}}{\Delta t}\right\|_{-1}\left\|\nabla \xi_{h}^{l}\right\|_{0} \\
& +C\left\|\frac{\hat{u}^{l-1}-\bar{u}^{l-1}}{\triangle t}\right\|_{0}\left\|\nabla \xi_{h}^{l}\right\|_{0}+C\left\|\frac{\hat{\xi}_{h}^{l-1}-\xi_{h}^{l-1}}{\Delta t}\right\|_{-1}\left\|\nabla \xi_{h}^{l}\right\|_{0} \\
& +\nu_{a d d}\left\|\nabla \eta_{h}^{l}\right\|_{0}\left\|\nabla \xi_{h}^{l}\right\|_{0}+\nu_{T}\left\|\left(I-P_{L_{H}}\right) \nabla u^{l}\right\|_{0}\left\|\left(I-P_{L_{H}}\right) \nabla \xi_{h}^{l}\right\|_{0} \\
& \leq C\left(\left\|\frac{u^{l}-\bar{u}_{h}^{l-1}}{\triangle t}-\nu \triangle u^{l}+\nabla p^{l}-f^{l}\right\|_{0}^{2}+\left\|\frac{\eta^{l}-\hat{\eta}_{h}^{l-1}}{\triangle t}\right\|_{-1}^{2}+\left\|\frac{\hat{u}^{l-1}-\bar{u}^{l-1}}{\Delta t}\right\|_{0}^{2}\right. \\
& \left.+\left\|\frac{\hat{\xi}_{h}^{l-1}-\xi_{h}^{l-1}}{\triangle t}\right\|_{-1}^{2}+\nu_{a d d}\left\|\nabla \eta_{h}^{l}\right\|_{0}^{2}+\nu_{T}\left\|\left(I-P_{L_{H}}\right) \nabla u^{l}\right\|_{0}^{2}\right)+\frac{\nu+\nu_{a d d}}{2}\left\|\nabla \xi_{h}^{l}\right\|_{0}^{2} .
\end{aligned}
$$

By (4.1), (4.10), (4.14) and (4.15), we get

$$
\begin{aligned}
& \frac{\left\|\xi_{h}^{l}\right\|_{0}^{2}-\left\|\xi_{h}^{l-1}\right\|_{0}^{2}}{\triangle t}+\left(\nu+\nu_{a d d}\right)\left\|\nabla \xi_{h}^{l}\right\|_{0}^{2} \leq C\left(\Delta t^{2}+h^{2 k+2}+\nu_{a d d} h^{2 k}\right. \\
& \left.+\frac{h^{2 k+2}}{\triangle t}\left\|\frac{\partial u}{\partial t}\right\|_{L^{2}\left(t_{l-1}, t_{l} ; H^{k}(\Omega)\right)}+\left\|\xi_{h}^{l-1}\right\|_{0}^{2}+\nu_{T}\left\|\left(I-P_{L_{H}}\right) \nabla u^{l}\right\|_{0}^{2}\right) .
\end{aligned}
$$

Summing inequality (4.16) from $i=1$ to $l$, we obtain

$$
\begin{aligned}
& \left\|\xi_{h}^{l}\right\|_{0}^{2}+\nu \triangle t \sum_{i=1}^{l}\left\|\nabla \xi_{h}^{i}\right\|_{0}^{2} \\
& \quad \leq C\left(\triangle t^{2}+h^{2 k+2}+\nu_{a d d} h^{2 k}+\nu_{T}\left\|\left(I-P_{L_{H}}\right) \nabla u^{l}\right\|_{0}^{2}+\triangle t \sum_{i=1}^{l-1}\left\|\xi_{h}^{i}\right\|_{0}^{2}\right) .
\end{aligned}
$$

Using discrete Gronwall's Lemma, we have

$$
\begin{aligned}
& \left\|\xi_{h}^{l}\right\|_{0}^{2}+\nu \triangle t \sum_{i=1}^{l}\left\|\nabla \xi_{h}^{i}\right\|_{0}^{2} \\
& \quad \leq C \exp (C T)\left(\triangle t^{2}+h^{2 k+2}+\nu_{a d d} h^{2 k}+\nu_{T}\left\|\left(I-P_{L_{H}}\right) \nabla u^{l}\right\|_{0}^{2}\right) .
\end{aligned}
$$

By triangle inequality, we deduce

$$
\left\|u_{h}^{l}\right\|_{\infty} \leq\left\|u_{h}^{l}-R_{h}^{l}\right\|_{\infty}+\left\|R_{h}^{l}\right\|_{\infty} .
$$

Using inverse inequality, $\left\|v_{h}\right\|_{\infty} \leq C h^{-1}\left\|\nabla v_{h}\right\|_{0}$ (see [4]), we get $\left\|u_{h}^{l}\right\|_{\infty} \leq C h^{-1}\left\|\nabla\left(u_{h}^{l}-R_{h}^{l}\right)\right\|_{0}+\left\|R_{h}^{l}\right\|_{\infty}$, $\triangle t\left\|u_{h}^{n+1}\right\|_{W^{1, \infty}} \leq \triangle t\left\|R_{h}^{l+1}\right\|_{W^{1, \infty}}+\triangle t\left\|\xi_{h}^{l+1}\right\|_{W^{1, \infty}} \leq C \triangle t+C h^{-1} \triangle t\left\|\xi_{h}^{l+1}\right\|_{1}$.

Using (4.17) and Lemma 4.4 we finish the proof. 
Theorem 1. Let $u_{h}^{n}$ be defined by (3.1) and $u$ be the solution of (1.1). Under the assumptions of Lemma 4 , for all $1 \leq n \leq N$ we have

$$
\begin{aligned}
& \left\|u^{n}-u_{h}^{n}\right\|_{0}^{2} \leq C \exp (C T)\left(\triangle t^{2}+h^{2 k+2}+\nu_{a d d} h^{2 k}+\nu_{T}\left\|\left(I-P_{L_{H}}\right) \nabla u^{n}\right\|_{0}^{2}\right), \\
& \nu \triangle t \sum_{i=1}^{n}\left\|\nabla\left(u^{n}-u_{h}^{n}\right)\right\|_{0}^{2} \leq C \exp (C T) \\
& \quad \times\left(\triangle t^{2}+h^{2 k+2}+\nu_{a d d} h^{2 k}+\nu_{T}\left\|\left(I-P_{L_{H}}\right) \nabla u^{n}\right\|_{0}^{2}\right)+C h^{2 k} .
\end{aligned}
$$

Proof. Using triangle inequality, (4.3) and (4.4), we obtain the desired result.

Corollary 1. Under the assumption of Lemma 4, Remark 3.2, the regularity assumption of $(u, p) \in\left(H^{3}(\Omega)^{2} \cap X, H^{2}(\Omega) \cap M\right) \forall t \in(0, T]$ and the assumption of $\nu_{T}$, for all $1 \leq n \leq N$ we have the following error analysis

$$
\begin{aligned}
& \left\|u^{n}-u_{h}^{n}\right\|_{0}^{2} \leq C \exp (C T)\left(\triangle t^{2}+h^{2(k+1)}\right), \\
& \nu \triangle t \sum_{i=1}^{n}\left\|\nabla\left(u^{n}-u_{h}^{n}\right)\right\|_{0}^{2} \leq C \exp (C T)\left(\triangle t^{2}+h^{2 k}\right), \quad k=1,2 .
\end{aligned}
$$

\subsection{Error estimate for pressure}

The following result on the pressure is a consequence of the previous theorem on the velocity.

Theorem 2. Under the assumptions of Lemma 4, for all $1 \leq n \leq N$ we have

$$
\left(\sum_{i=0}^{n} \triangle t\left\|p^{i}-p_{h}^{i}\right\|_{0}^{2}\right)^{1 / 2} \leq C\left(\triangle t+h^{k}+\nu_{T}\left\|\left(I-P_{L_{H}}\right) \nabla u_{h}^{n}\right\|_{0}\right),
$$

where $C$ is a positive constant independent of $\triangle t$ and $h$.

Proof. Multiplying (1.1) by $v_{h} \in X_{h}$ and subtracting the result form (3.6) with $\varphi_{h}=0$, we obtain

$$
\begin{aligned}
& d\left(Q_{h}^{n}-p_{h}^{n}, v_{h}\right)=-\left(\bar{e}(x, n), v_{h}\right)+d\left(Q_{h}^{n}-p^{n}, v_{h}\right)+\nu a\left(u_{h}^{n}-u^{n}, v_{h}\right) \\
& \quad+\left(\frac{\left(u^{n}-u_{h}^{n}\right)-\left(\hat{u}_{h}^{n-1}-\bar{u}_{h}^{n-1}\right)}{\triangle t}, v_{h}\right)+\nu_{T}\left(\left(I-P_{L_{h}}\right) \nabla u_{h}^{n},\left(I-P_{L_{H}}\right) \nabla v_{h}\right) .
\end{aligned}
$$

By the inf-sup condition and Cauchy-Schwarz inequality, we get

$$
\begin{aligned}
& \left\|Q_{h}^{n}-p_{h}^{n}\right\|_{0} \leq C\left(\|e(x, n)\|_{0}+\left\|\frac{u-u_{h}^{n}}{\triangle t}\right\|_{0}+\left\|\frac{\hat{u}_{h}^{n-1}-\bar{u}_{h}^{n-1}}{\triangle t}\right\|_{0}+\left\|Q_{h}^{n}-p^{n}\right\|_{0}\right) \\
& \quad+\nu\left\|\nabla\left(u_{h}^{n}-u^{n}\right)\right\|_{0}+\nu_{T}\left\|\left(I-P_{L_{H}}\right) \nabla u_{h}^{n}\right\|_{0} .
\end{aligned}
$$

Using (4.1), (4.10) and (4.18), we arrive at

$$
\left(\sum_{i=0}^{n} \Delta t\left\|Q_{h}^{i}-p_{h}^{i}\right\|_{0}^{2}\right)^{1 / 2} \leq C\left(\triangle t+h^{k}+\nu_{T}\left\|\left(I-P_{L_{H}}\right) \nabla u_{h}^{n}\right\|_{0}\right) .
$$


By triangle inequality, we have

$$
\begin{aligned}
\left(\sum_{i=0}^{n} \triangle t\left\|p^{i}-p_{h}^{i}\right\|_{0}^{2}\right)^{1 / 2} & \leq\left(\sum_{i=0}^{n} \triangle t\left\|Q_{h}^{i}-p_{h}^{i}\right\|_{0}^{2}\right)^{1 / 2}+\left(\sum_{i=0}^{n} \triangle t\left\|Q_{h}^{i}-p^{i}\right\|_{0}^{2}\right)^{1 / 2} \\
& \leq C\left(\triangle t+h^{k}+\nu_{T}\left\|\left(I-P_{L_{H}}\right) \nabla u_{h}^{n}\right\|_{0}\right) .
\end{aligned}
$$

Therefore, we finish the proof.

Corollary 2. Under the assumptions of Lemma 4 and Remark 3.2, the regularity assumption of $(u, p) \in\left(H^{3}(\Omega)^{2} \cap X, H^{2}(\Omega) \cap M\right) \forall t \in(0, T]$ and the assumption of $\nu_{T}$, we have the following error estimate

$$
\left(\sum_{i=0}^{n} \triangle t\left\|p^{i}-p_{h}^{i}\right\|_{0}^{2}\right)^{1 / 2} \leq C\left(\triangle t+h^{k}\right), \quad k=1,2
$$

\section{$5 \quad$ Numerical Results}

In this section, we present some numerical results to verify the theoretical results obtained in the previous section and show the effect of our methods. Here, we use the software package FreeFEM++ [21] for our program.

\subsection{Analytical solution problems}

In this subsection, we present some numerical results of the Navier-Stokes problems with the analytical solution

$$
\begin{aligned}
u_{1} & =10 x^{2}(x-1)(2 x-1) y^{2}(y-1)^{2} \exp \left(-2 \pi^{2} t \nu\right), \\
u_{2} & =-10 x(x-1)(2 x-1) y^{2}(y-1)^{2} \exp \left(-2 \pi^{2} t \nu\right), \\
p & =20(2 x-1)(2 y-1) \exp \left(-4 \pi^{2} t \nu\right) .
\end{aligned}
$$

The boundary and initial conditions in (1.1) are set equal to the analytical solution and $f$ is given by evaluating the momentum equation of the problem (1.1) for the analytical solution. We choose $\Delta t=0.001, T=1, \delta=0.5$ and $R e=2000$. We present the numerical results with different $h$ respectively by using Taylor-Hood element, and $L_{H}=\left\{\mathbb{L} \in\left(L^{2}(\Omega)\right)^{2 \times 2},\left.\mathbb{L}\right|_{K} \in P_{1 d c}^{2 \times 2} \forall K \in\right.$ $\left.\Im_{h}\right\}, P_{1 d c}$ means the piecewise linear discontinuous finite element space [16].

Table 1. The numerical results of MMOCVMS at $T=1$ with $\triangle t=0.001, R e=2000$, $\nu_{T}=\delta h^{2}\left\|\nabla u_{h}\right\|_{0}$.

\begin{tabular}{lcccllll}
\hline \multirow{2}{*}{$1 / h$} & $\frac{\left\|u-u_{h}\right\|_{0}}{\|u\|_{0}}$ & $\frac{\left\|\nabla\left(u-u_{h}\right)\right\|_{0}}{\|u\|_{0}}$ & $\frac{\left\|p-p_{h}\right\|_{0}}{\|p\|_{0}}$ & $u_{L_{2}}$ rate & $u_{H_{1}}$ rate & $P_{L_{2}}$ rate & CPU, s \\
\hline 10 & $2.59204 \mathrm{e}-3$ & $3.06620 \mathrm{e}-2$ & $7.74597 \mathrm{e}-3$ & - & - & - & 178.433 \\
20 & $3.14163 \mathrm{e}-4$ & $7.47865 \mathrm{e}-3$ & $1.93649 \mathrm{e}-3$ & 3.0445 & 2.0356 & 2.0000 & 727.026 \\
30 & $9.41846 \mathrm{e}-5$ & $3.29969 \mathrm{e}-3$ & $8.60663 \mathrm{e}-4$ & 2.9711 & 2.0180 & 2.0000 & 1650.72 \\
40 & $4.30740 \mathrm{e}-5$ & $1.85122 \mathrm{e}-3$ & $4.84123 \mathrm{e}-4$ & 2.7195 & 2.0091 & 2.0000 & 2988.12 \\
\hline
\end{tabular}


Table 2. The numerical results of MMOCFEM mixed finite element method at $T=1$ with $\triangle t=0.001, R e=2000$.

\begin{tabular}{llllllll}
\hline $1 / h$ & $\frac{\left\|u-u_{h}\right\|_{0}}{\|u\|_{0}}$ & $\frac{\left\|\nabla\left(u-u_{h}\right)\right\|_{0}}{\|u\|_{0}}$ & $\frac{\left\|p-p_{h}\right\|_{0}}{\|p\|_{0}}$ & $u_{L_{2}}$ rate & $u_{H_{1}}$ rate & $P_{L_{2}}$ rate & CPU, s \\
\hline 10 & $2.59204 \mathrm{e}-3$ & $3.06620 \mathrm{e}-2$ & $7.74597 \mathrm{e}-3$ & - & - & - & 214.547 \\
20 & $3.14163 \mathrm{e}-4$ & $7.47865 \mathrm{e}-3$ & $1.93649 \mathrm{e}-3$ & 3.0445 & 2.0356 & 2.0000 & 872.899 \\
30 & $9.41846 \mathrm{e}-5$ & $3.29969 \mathrm{e}-3$ & $8.60663 \mathrm{e}-4$ & 2.97105 & 2.01799 & 2.0000 & 1987.68 \\
40 & $4.3074 \mathrm{e}-5$ & $1.85122 \mathrm{e}-3$ & $4.84123 \mathrm{e}-4$ & 2.7195 & 2.0091 & 2.0000 & 3553.67 \\
\hline
\end{tabular}

Table 3. The numerical results of MMOCVMS at $T=1$ with $h=1 / 100, R e=2000$, $\nu_{T}=\delta h^{2}\left\|\nabla u_{h}\right\|_{0}$.

\begin{tabular}{lllllll}
\hline$\Delta t$ & $\frac{\left\|u-u_{h}\right\|_{0}}{\|u\|_{0}}$ & $\frac{\left\|\nabla\left(u-u_{h}\right)\right\|_{0}}{\|u\|_{0}}$ & $\frac{\left\|p-p_{h}\right\|_{0}}{\|p\|_{0}}$ & $u_{L_{2}}$ rate & $u_{H_{1}}$ rate & CPU, s \\
\hline 0.1 & $1.81239 \mathrm{e}-3$ & $2.23524 \mathrm{e}-2$ & $7.74605 \mathrm{e}-5$ & - & - & 207.386 \\
0.05 & $9.06667 \mathrm{e}-4$ & $1.16259 \mathrm{e}-3$ & $7.74599 \mathrm{e}-5$ & 1.13258 & 1.06939 & 376.132 \\
0.025 & $4.5537 \mathrm{e}-4$ & $6.46561 \mathrm{e}-4$ & $7.74597 \mathrm{e}-5$ & 0.9935 & 0.8464 & 752.062 \\
0.0125 & $2.28506 \mathrm{e}-4$ & $4.16179 \mathrm{e}-4$ & $7.74597 \mathrm{e}-5$ & 0.9774 & 0.6272 & 1525.39 \\
\hline
\end{tabular}

The numerical results in Table 1 and 2 show that the convergence rates are $\mathcal{O}\left(h^{3}\right)$ of the $L^{2}$-norm for $u, \mathcal{O}\left(h^{2}\right)$ of the semi $H^{1}$-norm for $u$ and $\mathcal{O}\left(h^{2}\right)$ of the $L^{2}$-norm for $p$, which agree very well with our theoretical results by using $P_{2}-P_{1}$ finite element spaces. In Table 3 and 4 , we present the convergence test results for time. We can see that the time convergence order is $\mathcal{O}(\Delta t)$, which agrees well with our theory analysis. Comparing our numerical results with the MMOCFEM's, we can see that the error and the convergence rates are very similar. But our method can save much time.

\subsection{The lid driven cavity problem}

In this subsection we show the numerical results of lid driven cavity problem. The two-dimensional lid driven was formulated as in $\Omega=(0,1)^{2}$, the boundary conditions are $u_{1}=1, u_{2}=0$ on the top lid and $u_{1}=0, u_{2}=0$ on the other lids. In a former work [5], it was suggested that the first Hopf bifurcation occurs around Reynolds number $\mathrm{Re}=7500$. Since then various results were given in the literature $[5,12,14]$. In this numerical experiments, $h=\frac{1}{60}, \Delta t=$ $0.01, \nu_{T}=\delta h^{2}\left\|\nabla u_{h}\right\|_{0}, \delta=0.5$ are chosen. We choose the Taylor-Hood element and $L_{H}=\left\{\mathbb{L} \in\left(L^{2}(\Omega)\right)^{2 \times 2},\left.\mathbb{L}\right|_{K} \in P_{1 d c}^{2 \times 2} \forall K \in \Im_{h}\right\}$.

Firstly, we choose $R e=5000$, which is a good choice as there are some comparisons available in the literature and as the steady solution is still stable but not too far from the first Hopf bifurcation. Figure 1 gives the numerical results, (a) the numerical streamline at $t=250$, (b) the numerical pressure contours at $t=250,(\mathrm{c})$ the computed $u$-velocity profiles along a vertical line passing through the geometric center of the cavity, (d) the computed $v$-velocity profiles along a horizontal line passing through the geometric center of the cavity, (e) the evolution of the kinetic energy in time and (f) the evolution of $\left\|u_{h}^{n}-u_{h}^{n-1}\right\|_{0} /\left\|u_{h}^{n}\right\|_{0}$ in time. 
Table 4. The numerical results of MMOCFEM at $T=1$ with $h=1 / 100, R e=2000$.

\begin{tabular}{lllllll}
\hline$\Delta t$ & $\frac{\left\|u-u_{h}\right\|_{0}}{\|u\|_{0}}$ & $\frac{\left\|\nabla\left(u-u_{h}\right)\right\|_{0}}{\|u\|_{0}}$ & $\frac{\left\|p-p_{h}\right\|_{0}}{\|p\|_{0}}$ & $u_{L_{2}}$ rate & $u_{H_{1}}$ rate & CPU, s \\
\hline 0.1 & $1.81239 \mathrm{e}-3$ & $2.23524 \mathrm{e}-2$ & $7.74605 \mathrm{e}-3$ & - & - & 211.911 \\
0.05 & $9.06667 \mathrm{e}-4$ & $1.16259 \mathrm{e}-3$ & $7.74599 \mathrm{e}-5$ & 1.13258 & 1.06939 & 385.82 \\
0.025 & $4.5537 \mathrm{e}-4$ & $6.46561 \mathrm{e}-4$ & $7.74597 \mathrm{e}-5$ & 0.9935 & 0.8464 & 757.553 \\
0.0125 & $2.28506 \mathrm{e}-4$ & $4.16179 \mathrm{e}-4$ & $7.74597 \mathrm{e}-5$ & 0.9774 & 0.6272 & 1536.96 \\
\hline
\end{tabular}

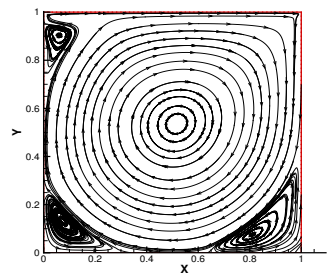

(a) The numerical streamline at $t=250$

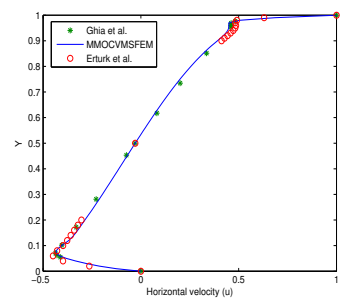

(d) The computed $v$-velocity

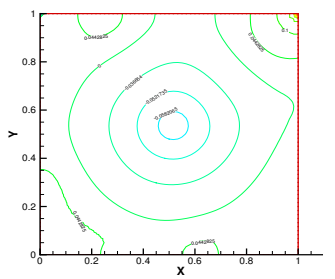

(b) The numerical pressure contours at $t=250$

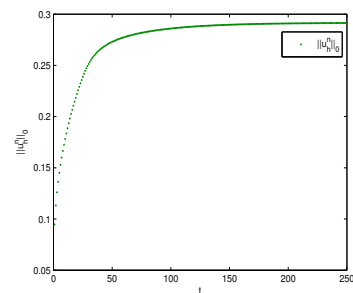

(e) Evolution of the kinetic energy in time

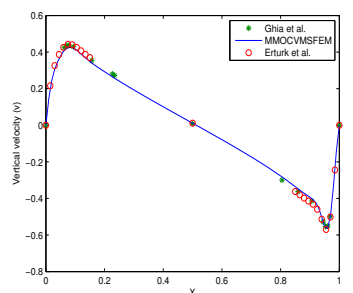

(c) The computed $u$-velocity

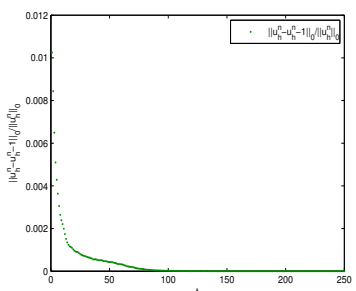

(f) The evolution of $\| u_{h}^{n}-$ $u_{h}^{n-1}\left\|_{0} /\right\| u_{h}^{n} \|_{0}$ in time

Figure 1. The numerical results for $R e=5000$ with $\nu_{T}=\delta h^{2}\left\|\nabla u_{h}\right\|_{0}$.

Figure 2 gives the numerical results for $R e=7500$, (a) the numerical streamline at $t=250,(\mathrm{~b})$ the numerical pressure contours at $t=250$, (c) the computed $u$-velocity profiles along a vertical line passing through the geometric center of the cavity, (d) the computed $v$-velocity profiles along a horizontal line passing through the geometric center of the cavity, (e) the evolution of the kinetic energy in time and (f) the evolution of $\left\|u_{h}^{n}-u_{h}^{n-1}\right\|_{0} /\left\|u_{h}^{n}\right\|_{0}$ in time.

From the numerical results, we can see that the kinetic energy reaches a stable state, $\left\|u_{h}^{n}-u_{h}^{n-1}\right\|_{0} /\left\|u_{h}^{n}\right\|_{0}$ changes very small. It means that we get a steady solution of the time-dependent Navier-Stokes equations. And, comparing with the results of the steady Navier-Stokes equations given by Ghia et al. [14] and Erturk et al. [12], we can see that $u$-velocity profiles along a vertical line passing through the geometric center of the cavity and $v$-velocity profiles along a horizontal line passing through the geometric center of the 


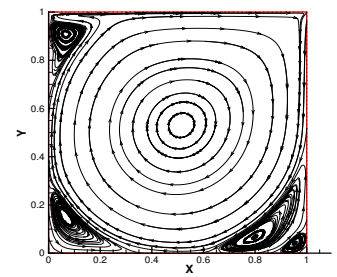

(a) The numerical streamline at $t=250$

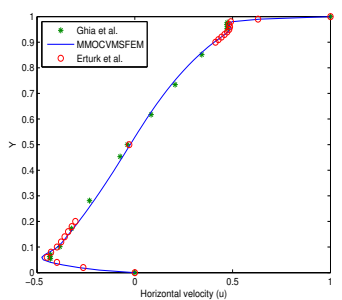

(d) The computed $u$-velocity

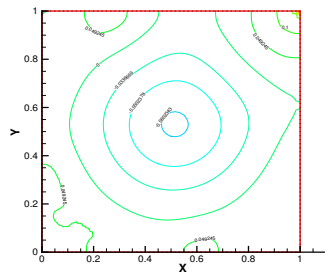

(b) The numerical pressure contours at $t=250$

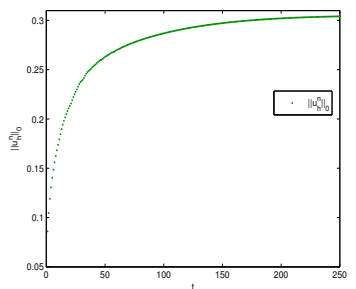

(e) The evolution of the kinetic energy in time

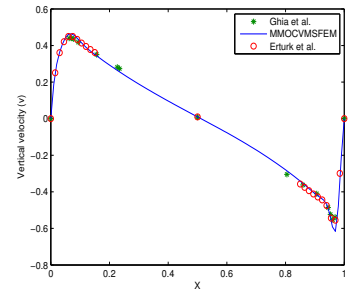

(c) The computed $v$-velocity

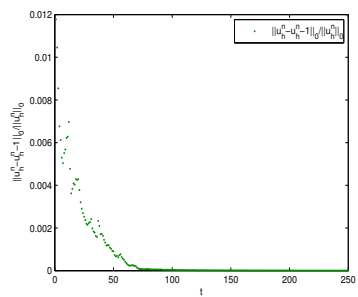

(f) The evolution of $\| u_{h}^{n}-$ $u_{h}^{n-1}\left\|_{0} /\right\| u_{h}^{n} \|_{0}$ in time

Figure 2. The numerical results for $R e=7500$ with $\nu_{T}=\delta h^{2}\left\|\nabla u_{h}\right\|_{0}$.

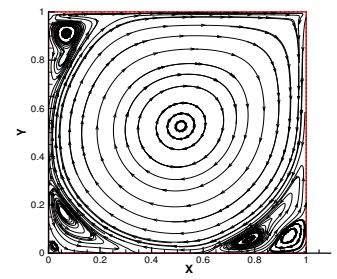

(a) $\mathrm{t}=180$

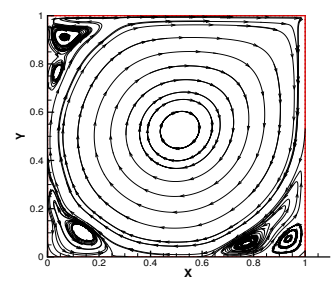

(d) $\mathrm{t}=240$

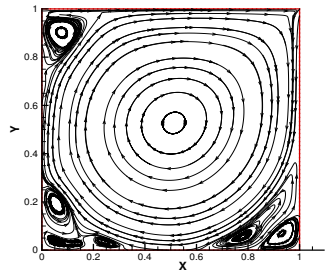

(b) $\mathrm{t}=200$

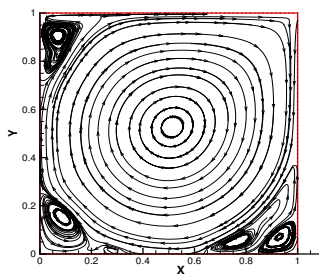

(e) $\mathrm{t}=260$

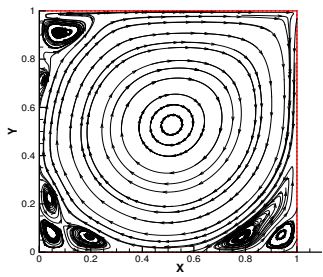

(c) $\mathrm{t}=220$

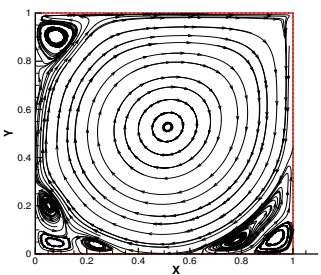

(f) $t=280$

Figure 3. The numerical velocity for $R e=10000$ with $\nu_{T}=\delta h^{2}\left\|u_{h}^{n}\right\|_{0}$ at different time. 


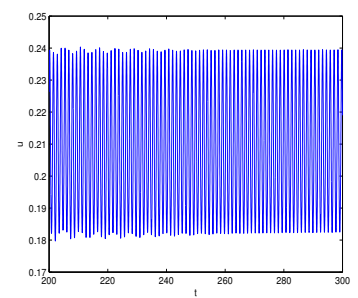

(a) $u$-velocity history

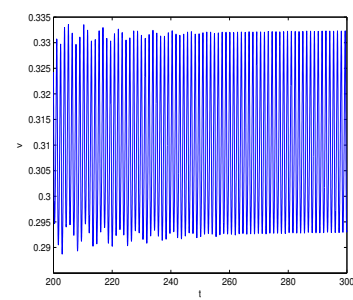

(b) $v$-velocity history

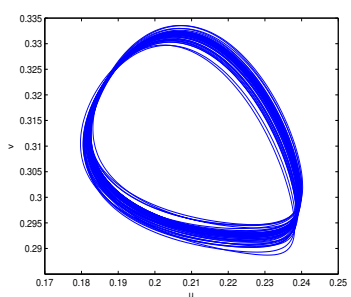

(c) Phase portrait

Figure 4. $u$-velocity history (a), $v$-velocity history (b) and the phase portrait (c), at monitoring point $(1 / 8,13 / 16)$ for $R e=10000$ with $\nu_{T}=\delta h^{2}\left\|u_{h}^{n}\right\|_{0}$ from $t=200$ to $t=300$.

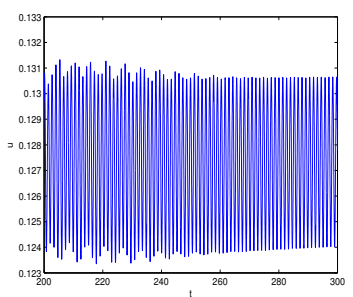

(a) $u$-velocity history

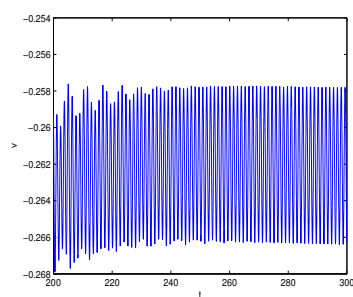

(b) $v$-velocity history

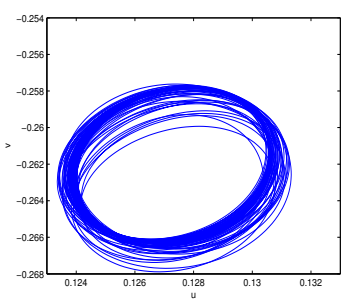

(c) Phase portrait

Figure 5. $u$-velocity history (a), $u$-velocity history (b) and the phase portrait (c), at monitoring point $(7 / 8,13 / 16)$ for $R e=10000$ with $\nu_{T}=\delta h^{2}\left\|u_{h}^{n}\right\|_{0}$ from $t=200$ to $t=300$.

cavity agree very well.

Finally, we show some numerical results for $R e=10000$, which is probably the most famous value and for quite a long time the question was to known if the steady solution was stable or not for this Reynolds number. In [6], Bruneau and Saad shown that the steady is not stable and the first Hopf bifurcation occurs around $R e=8000$. Figure 3 presents the streamlines at different time. We choose two monitoring points $(1 / 8,13 / 16)$ and $(7 / 8,13 / 16)$. Figures 4 and 5 show $u$-velocity history (a), $v$-velocity history (b) and the phase (c) portrait at monitoring points $(1 / 8,13 / 16)$ and $(7 / 8,13 / 16)$. It shows that the stable solution in mainly periodic with small variations in the amplitude of the time evolution at the monitoring points. And the phase portraits show that the variations in amplitude yield a solution which is quasi-periodic. Figure 6 shows the evolution of the kinetic energy in time (a) and evolution of $\| u_{h}^{n}-$ $u_{h}^{n-1}\left\|_{0} /\right\| u_{h}^{n} \|_{0}$ in time (b). We can see that the kinetic energy dose not change as the time change long enough and the errors don't change smaller. The results are very close the those shown in $[6]$. 


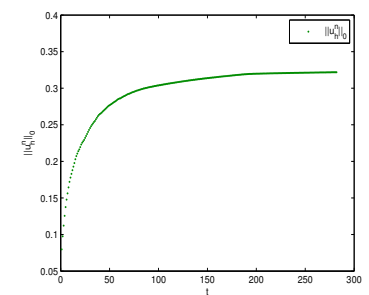

(a) The evolution of the kinetic energy in time

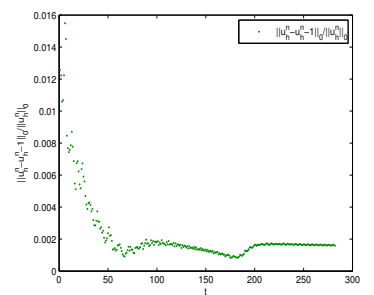

(b) The evolution of $\| u_{h}^{n}-$ $u_{h}^{n-1}\left\|_{0} /\right\| u_{h}^{n} \|_{0}$ in time

Figure 6. Evolution of the kinetic energy in time (a) and evolution of $\left\|u_{h}^{n}-u_{h}^{n-1}\right\|_{0} /\left\|u_{h}^{n}\right\|_{0}$ in time (b) for $R e=10000$ with $\nu_{T}=\delta h^{2}\left\|u_{h}^{n}\right\|_{0}$.

\subsection{The flow around a cylinder problems}

In this part, we investigate the two-dimensional under-resolved fluid flow around a cylinder [37], the physical model given by Figure 7. From Figure 7 we can see that, the height of the channel $H=0.41$ and the width $W=2.2$. The origin of the cylinder is at $(0.2,0.2)$, and the radius $R$ is equal to 0.05 . The time-dependent inlet flow velocity profiles are given by

$$
u_{1}=6 y(1-H) / H^{2}, \quad u_{2}=0 .
$$

The outlet boundary condition is given by

$$
\frac{\partial u}{\partial n}=0 .
$$

The boundary conditions of the two parallel planes and the cylinder surface are set as the non-slip boundary conditions. The Reynolds number is defined by $R e=2 \bar{U} R / \nu$ and $\bar{U}=2 u(0, H / 2) / 3$. Here, we choose $R e=100$, $\Delta t=0.01$. The finite element grid is presented by Figure 7 . We choose the Taylor-Hood element spaces. Figures 8 and 9 give the numerical results by the MMOCVMSFEM. Figures 10 and 11 present the numerical results by the MMOCFEM. From the numerical results, we can see that the numerical results of the MMOCVMSFEM agree well with the numerical results of MMOCFEM.

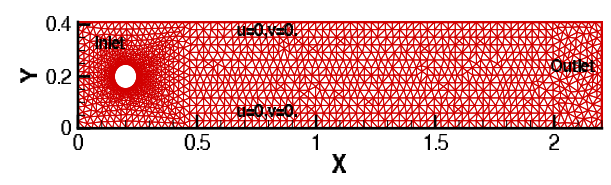

Figure 7. Physical model of the flow around a cylinder problems. 


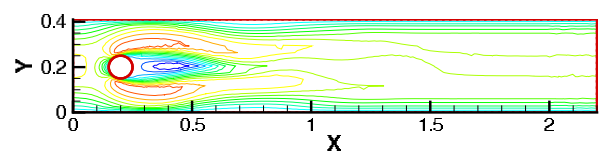

(a) $t=1$

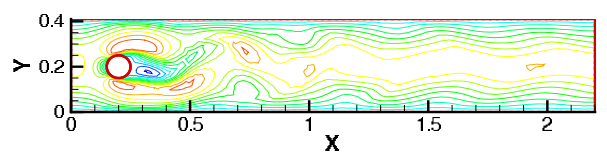

(b) $\mathrm{t}=5$

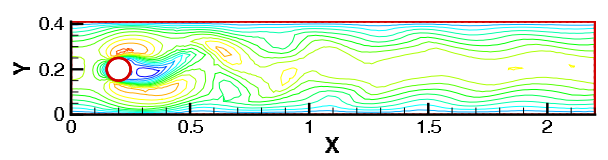

(c) $t=8$

Figure 8. The numerical contours of the velocity $u$ at different time by MMOCVMSFEM.

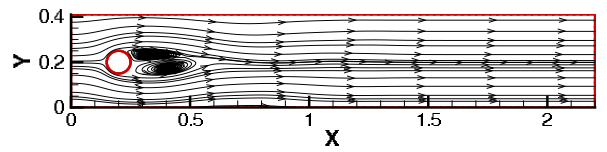

(a) $t=1$

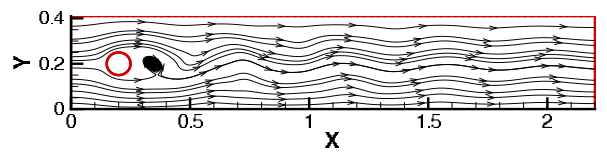

(b) $\mathrm{t}=5$

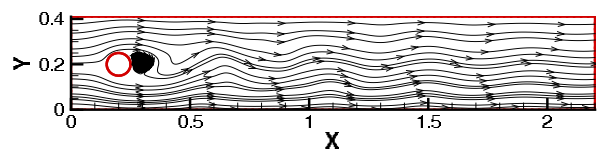

(c) $t=8$

Figure 9. The numerical streamlines at different time by MMOCVMSFEM.

\section{Conclusions}

In this work, we proposed a modified algorithm of characteristics variational multiscale finite element method for the time dependent Navier-Stokes equations. The theoretical analysis is discussed and illustrated. Error estimate shows that this method has a good convergence property. Numerical experiments are presented to demonstrate the high efficiency of the new algorithm. Also, further developments can extend these techniques and ideas to the general nonlinear problems and high-dimensional problems. 


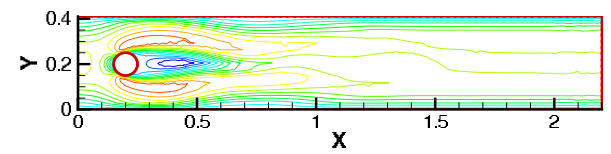

(a) $\mathrm{t}=1$

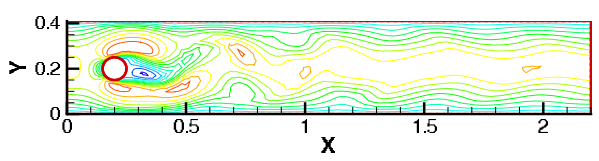

(b) $t=5$

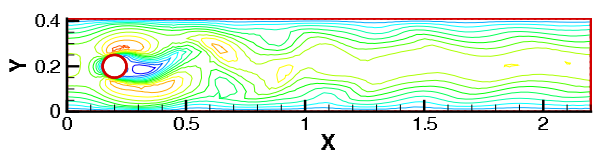

(c) $\mathrm{t}=8$

Figure 10. The numerical contours of the velocity $u$ at different time by MMOCFEM.

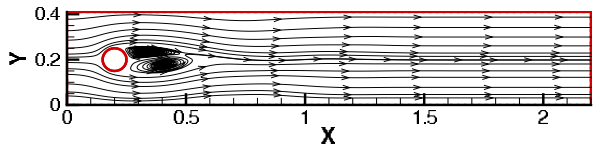

(a) $\mathrm{t}=1$

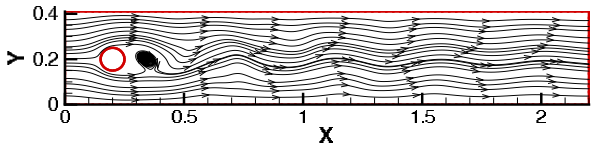

(b) $\mathrm{t}=5$

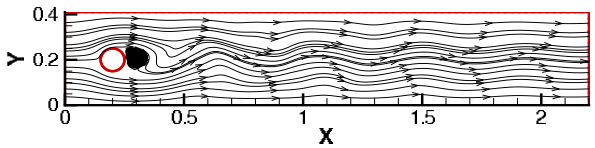

(c) $t=8$

Figure 11. The numerical streamlines at different time by MMOCFEM.

\section{Acknowledgements}

The authors would like to thank the anonymous referees for their valuable suggestions and comments, which helped to improve the quality of the paper.

\section{References}

[1] Y. Achdou and J.L. Guermond. Convergence analysis of a finite element projection/Lagrange-Galerkin method for the incompressible 
Navier-Stokes equations. SIAM J. Numer. Anal., 37(3):799-826, 2000. http://dx.doi.org/10.1137/S0036142996313580.

[2] T. Arbogast and M.F. Wheeler. A characteristics-mixed finite element method for advection-dominated transport problems. SIAM J. Numer. Anal., 32(2):404424, 1995. http://dx.doi.org/10.1137/0732017.

[3] R. Bermejo, P.G. del Sastre and L. Saavedra. A second order in time modified Lagrange-Galerkin finite element method for the incompressible Navier-Stokes equations. SIAM J. Numer. Anal., 50(6):3084-3109, 2012. http://dx.doi.org/10.1137/11085548X.

[4] K. Boukir, Y. Maday, B. MéTivet and E. Razafindrakoto. A highorder characteristics/finite element method for the incompressible NavierStokes equations. Int. J. Numer. Math. Fluids, 25(12):1421-1454, 1997. http://dx.doi.org/10.1002/(SICI)1097-0363(19971230)25:12<1421::AIDFLD334 $>3.0 . \mathrm{CO} ; 2-\mathrm{A}$.

[5] C.-H. Bruneau and C. Jouron. An efficient scheme for solving steady incompressible Navier-Stokes equations. J. Comput. Phys., 89(2):389-413, 1990. http://dx.doi.org/10.1016/0021-9991(90)90149-U.

[6] C.-H. Bruneau and M. Saad. The 2d lid-driven cavity problem revisited. Comput. Fluids, 35(3):326-348, 2006.

http://dx.doi.org/10.1016/j.compfluid.2004.12.004.

[7] G.C. Buscaglia and E.A. Dari. Implementation of the Lagrange-Galerkin method for the incompressible Navier-Stokes equations. Int. J. Numer. Methods fluids, 15(1):23-36, 1992. http://dx.doi.org/10.1002/fld.1650150103.

[8] S.S. Collis. Monitoring unresolved scales in multiscale turbulence modeling. Phys. Fluids, 13(6):1800-1806, 2001. http://dx.doi.org/10.1063/1.1367872.

[9] A. Corsini, F. Rispoli and A. Santoriello. A variational multiscale higher-order finite element formulation for turbomachinery flow computations. Comput. Methods Appl. Mech. Engrg., 194(45-47):4797-4823, 2005. http://dx.doi.org/10.1016/j.cma.2004.11.013.

[10] C.N. Dawson, T.F. Russell and M.F. Wheeler. Some improved error estimates for the modified method of characteristics. SIAM J. Numer. Anal., 26(6):14871512, 1989. http://dx.doi.org/10.1137/0726087.

[11] J. Douglas and T.F. Russell. Numerical methods for convection-dominated diffusion problems based on combing the method of characteristics with finite element or finite difference procedures. SIAM J. Numer. Anal., 19(5):871-885, 1982. http://dx.doi.org/10.1137/0719063.

[12] E. Erturk, T.C. Corke and C. Gökvöl. Numerical solutions of 2-D steady incompressible driven cavity flow at high Reynolds numbers. Int. J. Numer. Meth. Fluids, 48(7):747-774, 2005. http://dx.doi.org/10.1002/fld.953.

[13] R.E. Ewing and T.F. Russell. Multistep Galerkin method along characteristics for convection-diffusion problems. In H. Ammann and V.A. Solonnikov(Eds.), Adv. Comput. Methods P.D.E., Navier-Stokes Equations and Related Nonlinear Problems, pp. 28-36, Rutgers University, New Brunswick, NJ, 1981. IMACS.

[14] U. Ghia, K.N. Ghia and C.T. Shin. High-re solution for incompressible flow using the Navier-Stokes equations and a multigrid method. J. Comput. Phys., 48(3):387-411, 1982. http://dx.doi.org/10.1016/0021-9991(82)90058-4. 
[15] V. Girault and P.A. Raviart. Finite Element Method for Navier-Stokes Equations. Theory and Algorithms. Springer Series in Computational Mathematics. Springer-Verlag, Berlin, 1986.

[16] P. Hansbo. The characteristic streamline diffusion method for the timedependent incompressible Navier-Stokes equations. Comput. Methods Appl. Mech. Engrg., 99(2):171-186, 1992.

http://dx.doi.org/10.1016/0045-7825(92)90039-M.

[17] Y. He. A fully discrete stabilized finite-element method for the timedependent Navier-Stokes problem. IMA J. Numer. Anal., 23(4):665-691, 2003. http://dx.doi.org/10.1093/imanum/23.4.665.

[18] Y. He and J. Li. Convergence of three iterative methods based on the finite element discretization for the stationary Navier-Stokes equations. Comput. Methods Appl. Mech. Engrg., 198(15-16):1351-1359, 2009. http://dx.doi.org/10.1016/j.cma.2008.12.001.

[19] Y. He and W. Sun. Stability and convergence of the Crank-Nicolson/AdamsBashforth scheme for the time-dependent Navier-Stokes equations. SIAM J. Numer. Anal., 45(2):837-869, 2007. http://dx.doi.org/10.1137/050639910.

[20] Y. He and A. Wang. A simplified two-level method for the steady Navier-Stokes equations. Comput. Methods Appl. Mech. Engrg., 197(17-18):1568-1576, 2008. http://dx.doi.org/10.1016/j.cma.2007.11.032.

[21] F. Hecht, O. Pironneau and K. Ohtsuka. Freefem++, 2002. Available from Internet: http://www.freefem.org/ff++/. (Software Package)

[22] T.J.R. Hughes. Multiscale phenomena: Green's functions, the Dirichlet-toNeumann formulation, subgrid scale models, bubbles and the origins of stabilized methods. Comput. Methods Appl. Mech. Engrg., 127(1):387-401, 1995. http://dx.doi.org/10.1016/0045-7825(95)00844-9.

[23] T.J.R. Hughes, G.R. Feijóo, L. Mazzei and J. Quincy. The variational multiscale method-a paradigm for computational mechanics. Comput. Methods Appl. Mech. Engrg., 166(1):3-24, 1998. http://dx.doi.org/10.1016/S0045-7825(98)00079-6.

[24] T.J.R. Hughes, L. Mazzei, A. Oberai and A.A. Wray. The multiscale formulation of large eddy simulation: decay of homogeneous isotropic turbulence. Phys. Fluids, 13(2):505-512, 2001. http://dx.doi.org/10.1063/1.1332391.

[25] T.J.R. Hughes, A. Oberai, A. Assad and L. Mazzei. Large eddy simulation of turbulent channel flows by the variational multiscale method. Phys. Fluids, 13(6):1784-1799, 2001. http://dx.doi.org/10.1063/1.1367868.

[26] V. John and S. Kaya. A finite element variational multiscale method for the Navier-Stokes equations. SIAM J. Sci. Comp., 26(5):1485-1503, 2005. http://dx.doi.org/10.1137/030601533.

[27] V. John and S. Kaya. Finite element error analysis for a variational multiscale method for the Navier-Stokes equations. Adv. Comput. Math., 28(1):43-61, 2008. http://dx.doi.org/10.1007/s10444-005-9010-z.

[28] V. John, S. Kaya and A. Kindl. Finite element error analysis for a projectionbased variational multiscale method with nonlinear eddy viscosity. J. Math. Anal. Appl., 344(2):627-641, 2008. http://dx.doi.org/10.1016/j.jmaa.2008.03.015.

[29] V. John, S. Kaya and W. Layton. A two-level variational multiscale method for convection-dominated convection-diffusion equations. Comp. Meth. Appl. Math. Eng., 195(33-36):4594-4603, 2006.

http://dx.doi.org/10.1016/j.cma.2005.10.006. 
[30] V. John and A. Kindl. Numerical studies of finite element variational methods for turbulent flow simulations. Comput. Meth. Appl. Mech. Eng., 199(13-16):841852, 2010. http://dx.doi.org/10.1016/j.cma.2009.01.010.

[31] V. John and A. Kindl. A variational multiscale method for turbulent flow simulation with adaptive large scale space. J. Comput. Phys., 229(2):301-312, 2010.

[32] B. Koobus and C. Farhat. A variational multiscale method for the large eddy simulation of compressible turbulent flows on unstructured meshes-application to vortex shedding. Comput. Methods Appl. Mech. Eng., 193(15-16):1367-1383, 2004. http://dx.doi.org/10.1016/j.cma.2003.12.028.

[33] J. Li and $\mathrm{Z}$. Chen. A new local stabilized nonconforming finite element method for the Stokes equations. Computing, 82(2-3):157-170, 2008. http://dx.doi.org/10.1007/s00607-008-0001-z.

[34] O. Pironnequ. On the transport-diffusion algorithm and it's applications to the Navier-Stokes equations. Numer. Math., 38(3):309-332, 1982. http://dx.doi.org/10.1007/BF01396435.

[35] A. Quarteroni and A. Valli. Numerical Approximation of Partial Differential Equations. Springer-Verlag, Berlin, 1998. http://dx.doi.org/10.1007/978-3-540-85268-1.

[36] T.F. Russell. Time stepping along characteristics with incomplete iteration for a Galerkin approximation of miscible displacement in porous media. SIAM J. Numer. Anal., 22(5):970-1013, 1985. http://dx.doi.org/10.1137/0722059.

[37] M. Schäfer, S. Turek, F. Durst, E. Krause and R. Rannacher. Benchmark computations of laminar flow around a cylinder. In E.H. Hirschel(Ed.), Flow Simulation with High-Performance Computers II, Notes on Numerical Fluid Mechanics (NNFM), pp. 547-566. Vieweg+Teubner Verlag, Wiesbaden, 1996. http://dx.doi.org/10.1007/978-3-322-89849-4_39.

[38] Z. Si. Second order modified method of characteristics mixed defect-correction finite element method for time dependent Navier-Stokes problems. Numer. Algor., 59(2):271-300, 2012. http://dx.doi.org/10.1007/s11075-011-9489-y.

[39] Z. Si, Y. He and Y. Wang. Modified characteristics mixed defect-correction finite element method for the time-dependent Navier-Stokes problems. Appl. Anal., 94(4):701-724, 2015. http://dx.doi.org/10.1080/00036811.2014.899354.

[40] Z. Si, X. Song and P. Huang. Modified characteristics Gauge-Uzawa finite element method for time dependent conduction-convection problems. J. Sci. Comput., 58(1):1-24, 2014. http://dx.doi.org/10.1007/s10915-013-9721-0.

[41] Z. Si, J. Su and Y.N. He. A second order modified characteristics variational multiscale finite element method for time-dependent Navier-Stokes problems. J. Comp. Math., 31(2):154-174, 2013. http://dx.doi.org/10.4208/jcm.1210m3799.

[42] Z. Si, Y. Wang and S. Li. Decoupled modified characteristics finite element method for the time dependent Navier-Stokes/Darcy problem. Math. Method. Appl. Sci., 37(9):1392-1404, 2014. http://dx.doi.org/10.1002/mma.2901.

[43] E. Süli. Convergence and nonlinear stability of the Lagrange-Galerkin method for the Navier-stokes equations. Numer. Math., 53(4):459-483, 1988. http://dx.doi.org/10.1007/bf01396329.

[44] Y. Zhang and Y. He. Assessment of subgrid-scale models for the incompressible Navier-Stokes equations. J. Comput. Appl. Math., 234(2):593-604, 2010. http://dx.doi.org/10.1016/j.cam.2009.12.051. 